\title{
Technology Developments for a Beam Source of an NNBI System for DEMO
}

\author{
U. Fantz ${ }^{\mathrm{a}}$, C. Hopf ${ }^{\mathrm{a}}$, R. Friedl ${ }^{\mathrm{b}}$, S. Cristofaro ${ }^{\mathrm{a}}$, B. Heinemann ${ }^{\mathrm{a}}$, S. Lishev ${ }^{\mathrm{c}}$, A. Mimo $^{\mathrm{a}}$ \\ ${ }^{a}$ Max-Planck-Institut für Plasmaphysik, Boltzmannstr. 2, 85748 Garching, Germany \\ ${ }^{b}$ AG Experimentelle Plasmaphysik, Universität Augsburg, 86135 Augsburg, Germany \\ ${ }^{c}$ Faculty of Physics, Sofia University, 5 J. Bourchier Blvd., BG-1164 Sofia, Bulgaria
}

\begin{abstract}
Based on the requirements for heating and current drive of a DEMO (tokamak) device a neutral beam injection (NBI) system faces several challenges, from which the achievement of high wall-plug efficiency is the driving parameter for the beam line design. For boosting the wall-plug efficiency of the ITER NBI system from about 0.25 to target values around 0.4 (pulsed DEMO) or 0.5-0.6 (steady state DEMO), the standard gas neutralizer concept has to be modified or replaced by the innovative laser neutralizer concept for which the modular concept of the ion source allows for adaption. The technology developments of ITER's high power RF-driven ion source are continued by optimization of the RF-driver geometry and the resulting overlap of the driver plasmas in a large expansion chamber. Insights in the caesium evaporation and distribution in the ion source could allow for a reduction of the caesium consumption. On the other hand, the caesium dynamics influences the temporal source performance and the beam uniformity such that investigations on alternatives to caesium became an R\&D task. Finally, the status of proof-of-principle experiments on the laser neutralization technique and its challenges for upscaling from a lab scale experiment to a beam line are discussed.
\end{abstract}

Keywords: beam source, negative ions, neutral beam injection, DEMO.

\section{Introduction}

The European DEMO R\&D approach within the EUROfusion consortium follows the strategy of modest extrapolations from the ITER physics and technology basis to minimize development risks [1]. So far mostly two options have been studied, a pulsed tokamak (2 hours) based on rather conventional assumptions and a steady-state tokamak characterized by advanced physics and technology assumptions [2,3]. While the heating mix of DEMO will not be decided before 2024, it is clear that NBI is an attractive option due to its high current drive (CD) efficiency. Assuming NBI as the main heating and current drive (H\&CD) system, the flat-top NBI power requirements can be taken from scenario modeling [4]. Accordingly, about $50 \mathrm{MW}$ will be needed for the pulsed DEMO, whereas the steady-state device relies on an NBI power between 135 and $210 \mathrm{MW}$, depending on the specific scenario. A neutral beam injection system for a DEMO device has to be based on negative ions (NNBI), as it is the case for ITER [5,6,7]. A conceptual design of the beam line is being developed within the EUROfusion Work Package Heating and Current Drive WPHCD [8]. A key factor for the economics of any H\&CD system is its wall-plug efficiency, particularly if substantial CD is required. The present paper deals with the challenges and development needs that the wall-plug efficiency requirement implies for the beam source.

\section{Beam line requirements, concepts and priority development needs}

The required injected NBI power and beam energy are determined by the demands of the fusion device for heating and current drive. The beam energy regulates the position where the power is deposited in the plasma. Additionally it is a compromise between sufficient current drive efficiency, which increases with beam energy, and the risk of high voltage breakdowns. Reducing the beam energy from ITER's $1 \mathrm{MeV}$ to about $800 \mathrm{keV}$ could significantly improve voltage holding reliability at the costs of only $\sim 10 \%$ less CD efficiency.

Table 1. Requirements on the beam line (BL) for ITER [7] (with three injectors), a pulsed and a steady-state DEMO.

\begin{tabular}{|c|c|c|c|}
\hline & ITER & $\begin{array}{l}\text { DEMO } \\
\text { pulsed }\end{array}$ & $\begin{array}{c}\text { DEMO } \\
\text { steady-state }\end{array}$ \\
\hline \multicolumn{4}{|l|}{ Requirements } \\
\hline Beam energy & $1 \mathrm{MeV}$ & $0.8-1 \mathrm{MeV}$ & $\geq 0.8 \mathrm{MeV}$ \\
\hline Total injected power & $50 \mathrm{MW}$ & $50 \mathrm{MW}$ flat top & $135-210 \mathrm{MW}$ \\
\hline Number of BL & 3 & $2-3$ & $2-3$ \\
\hline Wall-plug efficiency & 0.28 & 0.4 & $0.5-0.6$ \\
\hline Subsystem efficiencie & & target values & target values \\
\hline Power supplies & 0.90 & 0.90 & 0.90 \\
\hline Acceleration & 0.70 & 0.80 & 0.85 \\
\hline Neutralization & 0.55 & 0.65 & 0.80 \\
\hline Duct transmission & 0.80 & 0.90 & 0.90 \\
\hline \multicolumn{4}{|c|}{ Source parameters per BL } \\
\hline Extracted current $\mathrm{D}^{-}$ & $57 \mathrm{~A}$ & $38-84 \mathrm{~A}$ & 74-204 A \\
\hline Eff. extraction area & $0.20 \mathrm{~m}^{2}$ & $0.19-0.28 \mathrm{~m}^{2}$ & $0.37-0.68 \mathrm{~m}^{2}$ \\
\hline Extr. current density & $286 \mathrm{~A} / \mathrm{m}^{2}$ & $200-300 \mathrm{~A} / \mathrm{m}^{2}$ & $200-300 \mathrm{~A} / \mathrm{m}^{2}$ \\
\hline \multicolumn{4}{|l|}{ Pulse length } \\
\hline Pulse length & $1 \mathrm{~h}$ & $2 \mathrm{~h}$ & $\infty$ \\
\hline
\end{tabular}

A comparison of the requirements for different devices is shown in table 1 . The main difference among the devices is the necessary wall-plug efficiency. For boosting the wall-plug efficiency of ITERs system from 0.28 up to 0.6 the neutralizer concept is the key. The advanced concept assumes the replacement of the 
standard gas neutralizer by an innovative laser neutralizer [8]. Although the physics of photon neutralization of negative deuterium ions by an intense laser beam is well understood, the challenge of realization on a large and fast negative ion beam is enormous [9] and the technological feasibility has not yet been demonstrated. On the other hand the advantages are enormous too: beside the theoretically unlimited neutralization efficiency - gas neutralizers are limited to $55 \%$ - they need no neutral feed gas, relaxing the demands on the pumping system and reducing the neutral background pressure. As a fallback option the combination of a gas neutralizer with energy recovery from the positive and negative residual ions is also under discussion [10]. A significant reduction of stripping losses in the accelerator as well as beamline and duct transmission losses by reducing the neutral gas background pressure contributes to enhancing the overall wall-plug efficiency and the neutral beam power.

The technology of the neutralizer as a spatially and functionally central component of the NNBI beamline has direct consequences for the source design. On top of that, the potentially higher power per beamline has to be matched by adapted source parameters. Using the reasonable assumption that the current density extracted from the ITER source represents an upper limit to what can be achieved at $0.3 \mathrm{~Pa}$ source pressure, the extracted current can be enhanced only by increasing the extraction area and the grid transparency. The attempt to increase current by increasing the extraction voltage would inevitably be accompanied by higher co-extracted electron current, which is not permissible due to associated heat load on the second grid.

\section{Beam source}

The size and geometry of the beam source(s) for one beam line is determined foremost by the choice of the type of the neutralizer such that the beam shape is ideal for the highest achievable neutralization efficiency. For the ITER beam line with the gas neutralizer one large source with rectangular area of $1 \times 2 \mathrm{~m}^{2}$ has been chosen $[5,7]$ whereas for an advanced DEMO concept with laser neutralizer a blade shaped beam would be ideal [8,11]. In both cases, the beam is focused by tilting the grid such that the beam fits through a duct cross section of about $0.7 \times 1 \mathrm{~m}^{2}$. The estimated impact on the breeding blanket is less than $1 \%$ for DEMO [4]. Furthermore, the arrangement of the drivers is a knob for improving the beam divergence - and thus the duct transmission through optimization of the plasma and beam uniformity.

\subsection{Modular concept}

The modular concept of the RF-driven ion source for the ITER beam line [12] allows optimal arrangement of the drivers at the back plate of the expansion chamber in order to illuminate the desired area of the first grid of the extraction system by a low temperature plasma (about 1 $\mathrm{eV}$ ). The prototype source for ITER [13] has one cylindrical driver with about $25 \mathrm{~cm}$ diameter (figure 1) connected to a rectangular expansion chamber $(0.3 \mathrm{~m}$ width, $0.6 \mathrm{~m}$ height, $0.2 \mathrm{~m}$ depth). The size scaling to the dimensions of the ITER source leads to 8 drivers in a $4 \times 2$ arrangement, where the RF coils of each horizontal pair are connected in series to one RF generator with an $\mathrm{RF}$ power of $200 \mathrm{~kW}$ at $1 \mathrm{MHz}$, resulting in $800 \mathrm{~kW}$ total RF power. An intermediate step in the European Roadmap for ITER NBI is the half-size ion source at ELISE $(2 \times 2$ drivers $)$ which has been operational at IPP since 2013 [13]. Only recently have the technological issues connected with high RF power operation been solved, allowing now exploration of the full parameter space [14]. The technological solutions and experience gained on ELISE are valuable input for the next step of the Roadmap, namely the sources at the European Neutral Beam Test Facility currently under construction in Padua [15]. First plasma of the ITER type ion source at the SPIDER test facility is expected in 2018 [16].

An increase of the RF efficiency and the RF reliability has been achieved by using state-of-the-art solid-state RF generators instead of tube based oscillators [14]. Moreover, at the prototype test facility BATMAN [13] the cylindrical driver has been replaced by a larger race-track-shaped driver (figure 1) that could replace two cylindrical drivers in future ion source concepts, as shown in figure 1 for the half-size source at ELISE. In this way it can be avoided to connect two drivers to one generator; furthermore the required RF power is reduced by about $30 \%$.

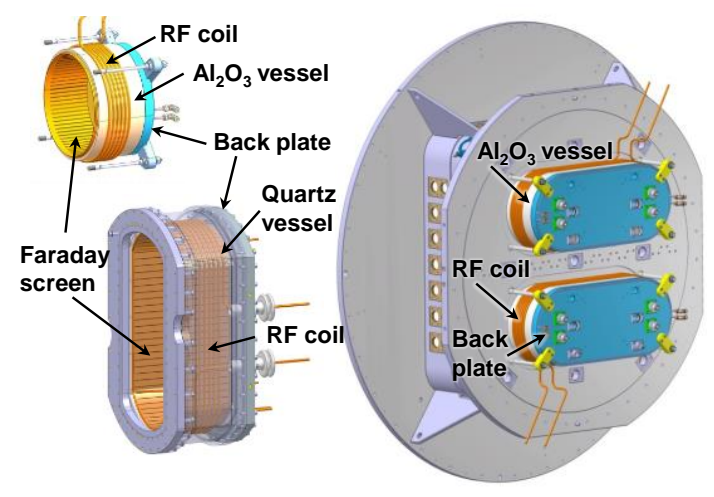

Fig. 1. Standard cylindrical driver, large race-track driver and a possible configuration of the modular concept for the half-size ITER source at the ELISE test facility (from left to right).

So far, one large expansion chamber with a certain depth is used in order to take advantage of the overlap of the expanding plasmas from the individual drivers, leading to a fairly homogeneous plasma close to the extraction area. The need for a magnetic filter field for electron reduction, however, results in a cross-B-drift. This effect is very pronounced in small sources and much less so in the half-size source [13]. In addition, the penetration of the magnetic field into the driver hampers the plasma flow out of the driver. For the purpose of studying the plasma flow in the source and improving plasma uniformity in front of the extraction system, a 2D fluid code was developed [17]. Figure 2 shows the geometry and the results obtained for the ion source on ELISE. The code takes into account the additional losses 
parallel to the magnetic field lines, $\mathbf{E} \times \mathbf{B}$ and diamagnetic drifts, and the power deposition by the RF coils.
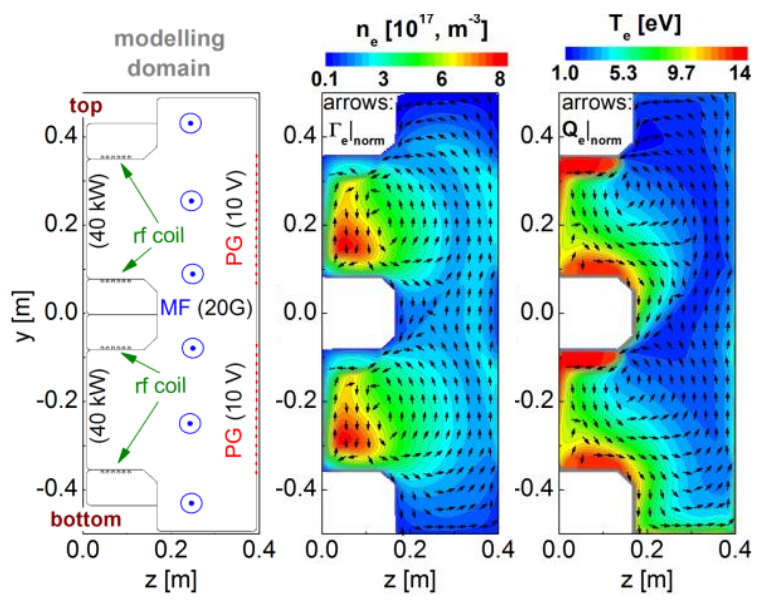

Fig. 2. Fluid simulations of the hydrogen plasma for a vertical cut along the drivers of ELISE calculated for an $\mathrm{RF}$ power of $40 \mathrm{~kW} /$ driver at $0.6 \mathrm{~Pa}$. Left: sketch of the geometry, center: electron density and particle flux, right: electron temperature and energy flux.

In the drivers an asymmetry of the electron density and temperature is seen. The electron flux and the energy flux (represented by arrows in the figure) show the cross-talk of the driver plasmas via the expansion chamber. This affects the accumulation of electrons in front of the two grid areas (PG), which is more pronounced at the side of the top drivers. That implies that the total electron current at the top part of the PG is a factor of 1.4 higher than in the bottom part. In order to avoid the cross-talk, one can think of dividing the common expansion chamber into individual chambers, each assigned to one, e.g. race-track-shaped, driver. This would also be in line with the idea of subdividing large sources into individual sub-sources [8] in order to increase redundancy. Another option would be using only horizontal walls which could also serve as additional caesium reservoirs in the source.

\subsection{Caesium management and alternatives}

According to the experience gained so far with large RF-driven ion sources, the long-pulse stability and reproducibility of the source performance is determined by how caesium is used to enhance negative ion generation $[12,13,18]$. At present caesium is evaporated into the source by ovens oriented either to side walls or the back-plate to build up reservoirs and to avoid Cs leakage into the accelerator as this can cause breakdowns. The evaporation rate is adjusted by the temperature of the liquid Cs reservoir. In the large sources more than one oven is used. Experiments and simulations of the Cs dynamics in these sources show that the position of the oven is not a critical issue [19] because Cs is efficiently redistributed by the plasma. Reservoirs build up at the inner source walls and act as Cs sources during plasma operation.

A major concern for long-pulse operation is the strong temporal decrease of the Cs content in the source
$[13,18]$ which results in a reduced Cs flux onto the plasma grid. Either the Cs reservoirs at the source walls are depleted too quickly or the caesium does not reach the grid. In fact, most of the caesium is ionized in the plasma phase and the positive bias potential applied to the grid system to suppress co-extracted electrons repels Cs ions. Simulations with the 3D Monte Carlo code CsFlow3D [20] support this hypothesis. Figure 3 shows the total Cs flux (neutrals and ions) to the plasma grid for the parameters of the half-size source at ELISE. Besides the redistribution by the plasma, sputtering of adsorbed $\mathrm{Cs}$ at the back-plate of the source by backstreaming positive ions created in the grid system is taken into account. This effect can be seen in the pulsed extraction case, which is the operation mode for ELISE (10 s extraction every $3 \mathrm{~min}$ ). The Cs flux is remarkably enhanced in these phases due to the fact that the sputtered particles have sufficient energy to cross the potential barrier. In fact, about $70 \%$ of the total Cs flux is carried by ions. The envelope of the curve shows that the Cs reservoirs are depleted over time.

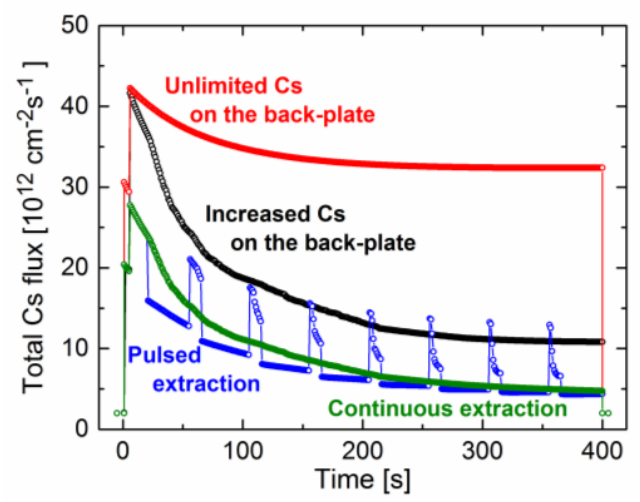

Fig. 3. Cs flux onto the plasma grid simulated with the CsFlow3D code for ELISE: pulsed extraction, continuous extraction and with increased and unlimited Cs reservoir at the back-plate of the source.

This depletion is similar for the simulated case assuming continuous extraction. Increasing the Cs reservoir on the back-plate of the source by a factor of four yields higher fluxes, but reveals depletion as well. Only assuming unlimited reservoirs at the back-plate enhances the total flux drastically and stabilizes it. Consequently, mechanisms have to be developed to either dedicatedly accelerate Cs ions to the grid or to increase the Cs reservoirs, most probably at the cost of increased Cs consumption.

The consumption of caesium determines the maintenance interval of the source as the ovens require regular refilling and thus replacement. The estimations for ITER and DEMO are based on the typical evaporation rate of $10 \mathrm{mg} \mathrm{h}^{-1}$ per oven [18,21], resulting in a consumption of $40 \mathrm{~g} \mathrm{yr}^{-1}$ for one beam line at ITER (100 d with 20 pulses, $400 \mathrm{~s}$ each) using three Cs ovens [22]. For DEMO, a consumption of 350-700 $\mathrm{g} \mathrm{yr}^{-1}$ with $80 \%$ availability throughout the year has been estimated using a similar source as for ITER [21]. By improving the Cs management at the testbed ELISE the evaporation 
rate has been reduced to typical values of less than $5 \mathrm{mg}$ $\mathrm{h}^{-1}$ from two ovens. Consequently, 100-200 $\mathrm{g} \mathrm{yr}^{-1}$ caesium would be sufficient for a DEMO beam line [18]. However, these estimations are based on operation in hydrogen, whereas it is well known that deuterium operation requires more caesium [13, 23]. The last deuterium campaign at ELISE [13] had an evaporation rate of at least a factor of two to five higher than in hydrogen for achieving stable currents for long pulses (1 h). This would result in a Cs consumption of about $0.5-1$ $\mathrm{kg} \mathrm{yr}^{-1}$ for DEMO, which is again very high. One should note that the critical issue is not the availability of the required amount of caesium, but the storage for a long period of time at a background pressure of $10^{-6} \mathrm{mbar}$ in the ion source, leading to contamination and inactivation of the chemically reactive caesium.

In order to increase the availability and reliability of a DEMO beam line it is highly desirable to reduce either the Cs consumption or to identify alternative materials. However, it should be kept in mind, that evaporation of fresh caesium has the major advantage that it reconditions deteriorated surfaces [13,23]. Fundamental investigations on the production mechanisms of negative ions at surfaces demonstrated that carbon-based materials such as HOPG (highly oriented purified graphite) or diamond-like coatings with or without boron doping could be promising materials [24]. Tantalum and tungsten are believed to increase volume production of negative ions by enhancing vibrational excitation of the hydrogen molecules. Other low work function materials, such as electron emitters used as cathode materials (e.g. $\mathrm{LaB}_{6}$ ), should also be considered, but they often require activation at high temperatures, typically above $1000^{\circ} \mathrm{C}$. This might not be feasible in an application as grid material, where the proper alignment of the large surfaces might be affected by the high temperature treatment. A compromise between completely replacing caesium and the need for Cs evaporation could be the usage of Cs-doped Mo. First investigations have been performed showing the proof-of-principle of $\mathrm{H}^{-}$surface formation at a maximum $\mathrm{Cs}$ surface concentration of below $10 \%$ so far [25].

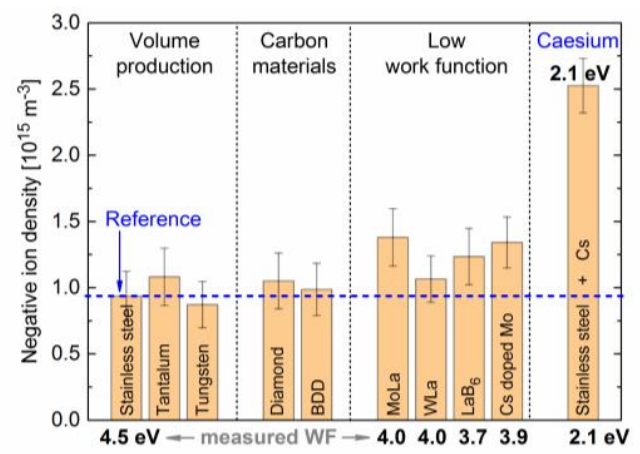

Fig. 4. Negative ion density measured above various surfaces in a hydrogen plasma [26]. The work function (WF), measured in a different setup [27], is assigned.

A comparative study of the materials under discussion was performed in a laboratory experiment in which plasma parameters close to the ones of the ion sources are achieved [26]. The results are summarized in figure 4. It shows the negative ion density in the plasma close to the respective material. As reference case in which the surface does not increase the negative ion density, stainless steel is used On the other hand, the target value is given by the density measured over the insitu Cs-coated surface. The work function of some of the materials was measured under similar conditions using the Fowler method [27].

An enhancement of the negative ion density above the surface is measured only for the low work function materials, although their performance is still well below the one of the caesiated surface. Moreover, all of the tested carbon materials showed clear indications of plasma-induced erosion already within plasma-on times of $10 \mathrm{~h}$, falling short of the demanded long-term stability for the use in ion sources. The work functions measured shortly after plasma treatment show that for caesium the work function of bulk $\mathrm{Cs}$ is achieved. However, the tested low work function materials have much higher values: $\mathrm{LaB}_{6}$ shows the lowest values of $3.7 \mathrm{eV}$, and $\mathrm{Cs}$ doped Mo, La-doped (0.7\%) Mo, and La-doped (1\%) W gave values of 3.9 or $4 \mathrm{eV}$. The temperature of the materials was $450{ }^{\circ} \mathrm{C}$ at maximum, which is much below the temperature at which for instance $\mathrm{LaB}_{6}$ becomes an effective electron emitter. This might explain the measured high work function and the small enhancement of the negative ion density. In conclusion, none of the tested materials so far comes close to the performance of a caesiated surface under ion source relevant conditions and temperatures. The focus should be laid on other low work function materials as well as on achieving higher Cs concentrations for Cs-doped Mo. For the latter the development of an in-situ implantation technique would be very beneficial. Finally, the ultimate test needs to be performed in a test facility with extraction system, since the purpose of the converter surface is not only to produce sufficient negative ions, but simultaneously to reduce the co-extracted electrons. At present, the latter issue actually limits the source performance [13].

\section{Proof-of-principle demonstration of a laser neutralizer}

Realization of laser neutralization for large beams faces several challenges: besides the huge beam cross section, neutralization at beam energies of around $1 \mathrm{MeV}$ requires very high optical powers. The required laser power is of the order of hundreds of megawatts (110 MW for a neutralization efficiency of $55 \%$, up to 400 MW for 95\% [9]) and can be achieved only by using optical cavities. Since the laser beam diameter is of the order of centimeters, the highly reflective mirrors have to withstand enormous optical power loads. To ensure that the neutron flux and possible plasma-surface interactions have no impact on the reflectivity, the mirrors have to be far away from the actual neutralization region. Together with the need for a sufficient laser beam cross section the cavity has to be very long, in the order of several tens of meters. Furthermore, decoupling from any vibrations is 
mandatory. Locking of the laser frequency to the cavity resonance is another demanding issue.

At present, proof-of-principle experiments are carried out in different laboratories in order to identify problems and potential show-stoppers (e.g. [28]). Also a full scale concept has been developed, the Siphore concept, for which first steps have already been started [11]. However, due to the manifold issues to be solved, the time scale of establishing such a sophisticated system at DEMO-relevant size is very long.

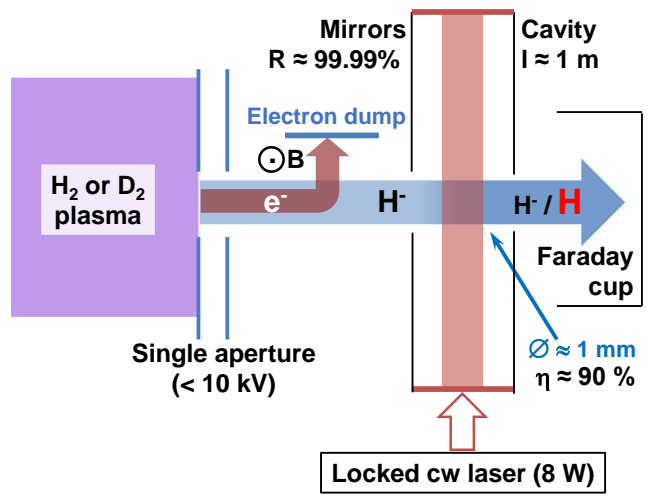

Fig. 5. Sketch of the laser neutralization test at a lab scale facility with one aperture for beam extraction.

At IPP a lab-scale experiment is set up aiming at the identification of principle issues in the coupling of the laser to the cavity and, further on, in the integration of the laser cavity in a low-power ion beam system. The envisaged scheme is shown in figure 5: negative ions and electrons are extracted from the plasma by a single aperture extraction system with less than $10 \mathrm{kV}$. Electrons are deflected by a magnetic field and negative ions are directed to the neutralization region that contains the optical cavity. The remaining negative current is measured by a Faraday cup. With an amplified optical power in the range of $10^{4}-10^{5} \mathrm{~W}$ within the cavity a neutralization degree of more than $90 \%$ is envisaged.

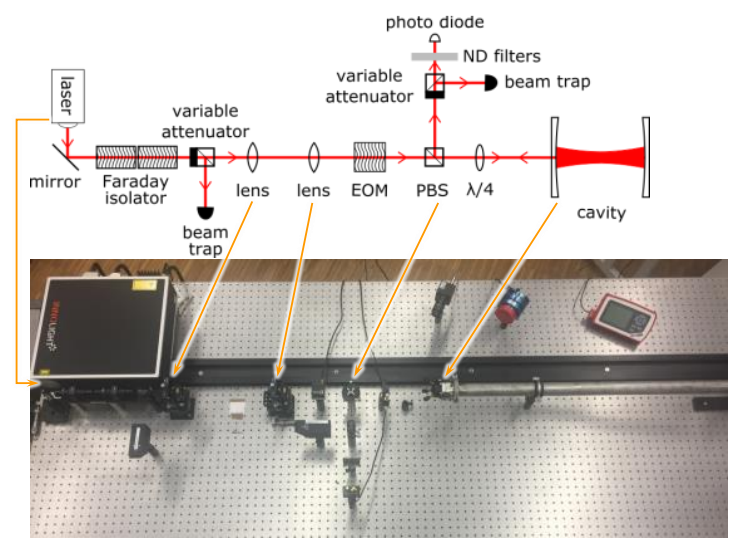

Fig. 6. Realization of the laser setup for the proof-of principle experiment.

Coupling of laser and cavity is tested in a separate setup first. Figure 6 shows the aligned optical setup on a vibration-isolated optical table. The cavity with a length of $1 \mathrm{~m}$ is located to the right. Laser light is led through optical isolators and a power regulation stage to the mode matching lenses which adjust the beam shape to the envisaged propagation within the cavity. The subsequent components are required to produce a feedback signal from the cavity to the laser head to continuously adapt the laser frequency to the cavity resonance (Pound-Drever-Hall locking scheme [29]) such that a stable lock is achieved. At present, last steps are under way to stabilize the lock before evacuating the cavity tube and testing the setup under the influence of vibrations from vacuum pumps. The next step will be the installation at a negative ion beam (figure 5).

In order to achieve reasonable neutralization at higher beam energies, the interaction region needs to be increased in a next stage, from currently around $1 \mathrm{~mm}$ to reasonable sizes of around $1 \mathrm{~cm}$ with or without refolding of the cavity.

\section{Conclusion}

The developments of the beam source for an NNBI system for DEMO are driven by the demand on achieving a reasonable wall-plug efficiency of the injector. Laser neutralization is the most promising option, but the concept is far from realization. First proof-of-principle experiments have started but large scale $R \& D$ projects are mandatory. Alternatively, the gas neutralizer with energy recovery may lead to efficiencies of about $40 \%$ which could be acceptable for a pulsed device for which the required power for heating and modest current drive is around $50 \mathrm{MW}$. The modular concept of the RF-driven ion source is well advanced and flexible enough to allow for adaption to the neutralizer and to the demands on plasma uniformity and beam homogeneity. RF efficiency and reliability have made huge progress towards their use for a DEMO beam line. Issues concerning the ion source are long-pulse stability and reproducibility, which depends strongly on the caesium management. The experiments at test facilities are accompanied by modeling activities. Reduction of caesium consumption or finding alternatives to caesium are topics of high priority for reliable negative ion production.

\section{Acknowledgments}

This work has been carried out within the framework of the EUROfusion Consortium and has received funding from the Euratom research and training programme 2014-2018 under grant agreement No 633053. The views and opinions expressed herein do not necessarily reflect those of the European Commission.

The authors would like to thank explicitly the NBI contributors of the WPHCD package of EUROfusion, in particular P. Sonato, A. Simonin, T. Franke and M.Q. Tran for many fruitful discussions.

\section{References}

[1] G.. Federici, DEMO Design Activity in Europe: Progress and Updates, Fusion Eng. Des. submitted this conference. 
[2] G. Federici et al., Fusion Eng. Des. 89 (2014) 882.

[3] R. Wenninger et al., Nucl. Fusion 57 (2017) 016011.

[4] G. Giruzzi et al., Nucl. Fusion 55 (2015) 073002.

[5] R.H. Hemsworth et al., Rev. Sci. Instrum. 67 (1996) 1120.

[6] T. Inoue et al., Fusion Eng. and Des. 56-57 (2001) 517.

[7] M. J. Singh et al., New J. Phys. 19 (2017) 055004.

[8] P. Sonato et al., Nucl. Fusion 57 (2017) 056026.

[9] M. Kovari and B. Crowley, Fusion Eng. Des. 85 (2010) 745.

[10] R. McAdams, AIP Conf. Proc 1515 (2013) 559.

[11] A. Simonin et al., Nucl. Fusion 55 (2015) 123020.

[12] R. S. Hemsworth et al., New J. Phys. 19 (2017) 025005.

[13] B. Heinemann et al., New J. Phys. 19 (2017) 015001.

[14] B. Heinemann et al., Latest achievements of the negative ion beam test facility ELISE, submitted this conference.

[15] V. Toigo et al., Nucl. Fusion 55 (2015) 083025

[16] V. Toigo et al., Nucl. Fusion 57 (2017) 086027.

[17] S. Lishev et al., AIP Conf. Proc. 1869 (2017) 030042.

[18] U. Fantz et al., Nucl. Fusion 57 (2017) 116007.

[19] A. Mimo et al., AIP Conf. Proc. 1869 (2017) 030019.

[20] R. Gutser, D. Wünderlich, and U. Fantz, Plasma Phys. Control. Fusion 53 (2011) 105014.

[21] P. Franzen and U. Fantz, Fusion Eng. Des. 89 (2014) 2594.

[22] R. Hemsworth et al., Nucl. Fusion 49 (2009) 045006.

[23] U. Fantz et al., Rev. Sci. Instrum. 87 (2016) $02 B 307$.

[24] G. Cartry et al., Phys. Plasmas 19 (2012) 063503.

[25] L. Schiesko et al., J. Appl. Phys. 118 (2015) 073303.

[26] U. Kurutz, R. Friedl and U. Fantz, Plasma Phys. Control. Fusion 59 (2017) 075008.

[27] R. Friedl and U. Fantz, J. Appl. Phys. 122 (2017) 083304.

[28] D. Bresteau, C. Blondel, and C. Drag, Rev. Sci. Instrum. 88 (2017) 113103.

[29] R. Drever et al., Appl. Phys. B 31 (1983) 97. 